\title{
Medical and surgical interventions and outcomes for infants with trisomy 18 (T18) or trisomy 13 (T13) at children's hospitals neonatal intensive care units (NICUs)
}

\author{
Krishna Acharya $\mathbb{1}^{1,2} \cdot$ Steven R. Leuthner ${ }^{1,2} \cdot$ Isabella Zaniletti $\mathbb{C}^{3} \cdot$ Jason Z. Niehaus $\mathbb{1}^{4,5} \cdot$ Christine E. Bishop ${ }^{6,7} \cdot$ \\ Carl H. Coghill $\mathbb{1}^{8,9}$ - Ankur Datta ${ }^{10,11} \cdot$ Narendra Dereddy ${ }^{12,13} \cdot$ Robert DiGeronimo $^{14,15} \cdot$ Laura Jackson $^{6,7}$. \\ Con Yee Ling ${ }^{16,17} \cdot$ Nana Matoba $\mathbb{1}^{10,11} \cdot$ Girija Natarajan $^{18,19} \cdot$ Sujir Pritha Nayak ${ }^{20,21}$ - Amy Brown Schlegel ${ }^{22,23}$. \\ Jamie Seale ${ }^{24}$ - Anita Shah ${ }^{25,26}$ - Julie Weiner ${ }^{27,28}$ • Helen O. Williams ${ }^{29,30}$ • Monica H. Wojcik $\mathbb{1}^{31,32}$. \\ Jessica T. Fry $\mathbb{1}^{10,11} \cdot$ Kevin Sullivan $\mathbb{1}^{33,34} \cdot$ on behalf of the Palliative Care and Ethics Focus Group of the Children's \\ Hospital Neonatal Consortium (CHNC)
}

Received: 25 January 2021 / Revised: 6 May 2021 / Accepted: 18 May 2021 / Published online: 10 June 2021

(C) The Author(s), under exclusive licence to Springer Nature America, Inc. 2021

\begin{abstract}
Objectives To examine characteristics and outcomes of T18 and T13 infants receiving intensive surgical and medical treatment compared to those receiving non-intensive treatment in NICUs.

Study design Retrospective cohort of infants in the Children's Hospitals National Consortium (CHNC) from 2010 to 2016 categorized into three groups by treatment received: surgical, intensive medical, or non-intensive.

Results Among 467 infants admitted, 62\% received intensive medical treatment; $27 \%$ received surgical treatment. The most common surgery was a gastrostomy tube. Survival in infants who received surgeries was $51 \%$; intensive medical treatment was $30 \%$, and non-intensive treatment was $72 \%$. Infants receiving surgeries spent more time in the NICU and were more likely to receive oxygen and feeding support at discharge.

Conclusions Infants with T13 or T18 at CHNC NICUs represent a select group for whom parents may have desired more intensive treatment. Survival to NICU discharge was possible, and surviving infants had a longer hospital stay and needed more discharge supports.
\end{abstract}

\section{Introduction}

Recent studies have added to our understanding of the medical care received by infants with trisomy 13 (T13) or trisomy 18 (T18) during and after the neonatal period [1-3]. Some infants with $\mathrm{T} 13$ or $\mathrm{T} 18$ receive interventions in neonatal intensive care units (NICUs), and their survival to NICU discharge has been reported to be between 20-60\% depending on risk factors [2]. The experience of families living with children with these conditions has also been described, and has emphasized the importance of

Supplementary information The online version contains supplementary material available at https://doi.org/10.1038/s41372021-01111-9.

Krishna Acharya

kkacharya@mcw.edu

Extended author information available on the last page of the article individualizing counseling to families based on both the specific medical co-morbidities associated with the genetic diagnoses and parental goals for their children [4-8].

After receiving a prenatal diagnosis of $\mathrm{T} 13$ or $\mathrm{T} 18$, some expectant families choose to carry to term and provide supportive care focused on comfort following delivery. These infants are often cared for in newborn nurseries and avoid NICU admission. Infants with T13 or T18 who are admitted to NICUs may receive interventions for various indications. These include parental desire for providing therapies to infants with confirmed diagnoses, interventions provided in the absence of a confirmatory diagnosis, or inherent medical instability while diagnosis is pending. Both $\mathrm{T} 13$ and $\mathrm{T} 18$ are associated with anomalies that can be emergently life threatening, but not all infants with $\mathrm{T} 13$ or $\mathrm{T} 18$ have these anomalies, and this makes caring for these infants in the immediate newborn period complex. Some infants do not require many neonatal interventions and survive, some survive after receiving interventions, and some die despite potentially life-sustaining medical 
or surgical interventions [9]. What is less well-described in the literature is a comparison of the outcomes and predictors of mortality among infants based on the level of medical and/or surgical interventions received.

In a cohort of infants admitted to US children's hospitals NICUs, our objectives were to: (1) categorize infants into groups based on level of NICU interventions received: nonintensive medical interventions, intensive medical interventions alone, and both intensive medical and surgical interventions; (2) assess demographic characteristics, NICU hospital course and discharge outcomes of infants in these groups; and (3) compare the characteristics of infants who die vs. survive to assess predictors of mortality in each of these groups. We hypothesized that infants with T13 or T18 admitted to US children's hospitals NICUs commonly undergo medical and surgical interventions and that these interventions have become more frequent over time.

\section{Materials and methods}

The Children's Hospitals Neonatal Database (CHND) captures clinical data for infants admitted to 34 US children's hospitals NICUs (the Children's Hospital Neonatal Consortium, or CHNC) until they either leave the NICU setting or achieve one year of age [10]. Greater than $50 \%$ of all infants represented in CHND are $>=37$ weeks gestation, and a quarter of those have surgical anomalies. The majority of infants in this cohort are out born. The database contains detailed information about medical and surgical interventions, comorbid diagnoses, prenatal and perinatal data, as well as the end of life care practices for infants who die before NICU discharge. Diagnoses of T13 or T18, when entered by a provider in a patient's chart, are uniquely recorded. For both, timing of diagnosis, whether prenatal or postnatal, is recorded. All data points are extracted by trained data coordinators at each CHND site through patient chart review. The CHND does not capture data on infants cared for exclusively in cardiac ICUs. All participating sites in CHND obtained Institutional Review Board approval for participation in the database. The database is approved for use by a central Institutional Review Board (IRB).

For this study, we included all infants with a diagnosis of T13 or T18 admitted to CHNC NICUs between 2010 and 2016. Mosaic T13 or T18 are typically included as a diagnosis of T13 or T18 by the data coordinators and not separately coded as mosaicism. We excluded readmissions as well as open charts, which reflect infants receiving NICU care at the end of the study period.

We first categorized infants into groups based on the level of NICU treatment they received: (1) surgical treatment included infants who received one or more major surgical procedures during their NICU stay; these infants may have received other intensive medical treatment as in (2); (2) intensive medical treatment included infants who received one or more of the following medical interventions: delivery room resuscitation requiring positivepressure ventilation, intubation, cardiac compressions or epinephrine, or in-NICU interventions to include noninvasive positive pressure respiratory support (defined as high flow nasal cannula $>2$ liters per minute or nasal CPAP), mechanical ventilation, surfactant, systemic steroids, vasopressors or inotropes, or inhaled nitric oxide (iNO); (3) non-intensive treatment included infants who received NICU care and none of these above interventions.

For surgical procedures, we obtained a list of every procedure performed and manually reviewed the list. Commonly performed NICU procedures, such as umbilical catheterizations, intubations, circumcisions, chest tube placement or abdominal paracentesis were not included in the list of surgical procedures. Major surgeries included were typically those that would require a trip to the operating room and/or presence of anesthesiology, such as bronchoscopy for airway evaluation, gastrostomy tube placement, repair of a diaphragmatic hernia, etc. Surgical procedures performed in infants with T13 or T18 and included in this study are listed in Appendix A (online).

Next, we assessed demographic characteristics for infants in each group, such as birth weight, NICU interventions received, details of hospital course, such as length of stay (LOS), and discharge outcomes. We assessed support provided to infants who survived to NICU discharge, including home oxygen or tube feeds. Finally, we compared the characteristics of infants who died vs. survived in each of these treatment groups.

Data analyses were performed using SAS Enterprise Guide 7.1 (Cary, NC). Categorical variables were compared using chi-squared tests of proportions or 2-sided Fisher's exact test as appropriate. Continuous variables were compared using non-parametric Wilcoxon rank-sum test and Kruskal-Wallis test, as distributions departed from normality. Multivariable logistic regression analysis was used to identify predictors of mortality in infants who received intensive medical or surgical treatment.

\section{Results}

Table 1 shows demographic characteristics of the cohort by treatment group. 150 infants had a diagnosis of T13 and 317 with T18. The majority of infants in this cohort received some degree of medical and/or surgical interventions (90\%). A third of patients in the cohort were prenatally diagnosed, and most were referred in from birth hospitals. Infants in the non-intensive treatment group were more likely to be fullterm, delivered vaginally, inborn, and have a 1-min Apgar score of $>7$. The proportion of prenatally diagnosed cases 
Table 1 Demographic characteristics of infants with T13 or T18 in 3 treatment groups $(n=467)$.

\begin{tabular}{|c|c|c|c|c|c|}
\hline & $\begin{array}{l}\text { Entire cohort } \\
467\end{array}$ & $\begin{array}{l}\text { Surgical } \\
\text { treatment } \\
129\end{array}$ & $\begin{array}{l}\text { Intensive medical } \\
\text { treatment } \\
292\end{array}$ & $\begin{array}{l}\text { Non-intensive } \\
\text { treatment } \\
46\end{array}$ & $p$-value \\
\hline Gestational age at birth (weeks) & $n(\%)$ & $n(\%)$ & $n(\%)$ & $n(\%)$ & 0.009 \\
\hline$\leq 27+6 / 7$ & $5(1 \%)$ & $2(2 \%)$ & $3(1 \%)$ & $0(0 \%)$ & \\
\hline 28 to $31+6 / 7$ & $41(9 \%)$ & $7(5 \%)$ & $32(11 \%)$ & $2(4 \%)$ & \\
\hline 32 to $36+6 / 7$ & $154(33 \%)$ & $53(41 \%)$ & $94(32 \%)$ & $7(15 \%)$ & \\
\hline$\geq 37$ & $267(57 \%)$ & $67(52 \%)$ & $163(56 \%)$ & $37(81 \%)$ & \\
\hline $\begin{array}{l}\text { Age at admission (days) } \\
\text { median (IQR) }\end{array}$ & $1[0,4]$ & $1[0,22]$ & $0[0,2]$ & $1[0,14]$ & $<0.001$ \\
\hline \multicolumn{6}{|l|}{ Birth weight (g) } \\
\hline$<=1500 \mathrm{~g}$ & $100(22 \%)$ & $25(20 \%)$ & $69(24 \%)$ & $6(13 \%)$ & 0.127 \\
\hline $1501-2499 \mathrm{~g}$ & $259(56 \%)$ & $81(64 \%)$ & $150(52 \%)$ & $28(62 \%)$ & \\
\hline$>=2500 \mathrm{~g}$ & $101(22 \%)$ & $21(16 \%)$ & $69(24 \%)$ & $11(25 \%)$ & \\
\hline Small-for-gestational age & $305(65 \%)$ & $91(70 \%)$ & $180(62 \%)$ & $34(74 \%)$ & 0.091 \\
\hline Male gender & $190(41 \%)$ & $52(40 \%)$ & $123(42 \%)$ & $15(33 \%)$ & 0.472 \\
\hline \multicolumn{6}{|l|}{ Maternal race ${ }^{\mathrm{a}}$} \\
\hline White & $241(52 \%)$ & $67(52 \%)$ & $152(52 \%)$ & $22(48 \%)$ & 0.244 \\
\hline Black & $119(25 \%)$ & $35(27 \%)$ & $73(25 \%)$ & $11(24 \%)$ & \\
\hline Other & $65(14 \%)$ & $17(13 \%)$ & $41(14 \%)$ & $7(15 \%)$ & \\
\hline Asian & $10(2 \%)$ & $1(1 \%)$ & $9(3 \%)$ & $0(0 \%)$ & \\
\hline Hawaiian/Pacific Islander & $4(1 \%)$ & $0(0 \%)$ & $2(1 \%)$ & $2(4 \%)$ & \\
\hline Inborn & $58(12 \%)$ & $11(8 \%)$ & $38(13 \%)$ & $9(19 \%)$ & 0.036 \\
\hline \multicolumn{6}{|l|}{ Delivery type } \\
\hline Cesarean & $278(60 \%)$ & $80(62 \%)$ & $182(62 \%)$ & $16(35 \%)$ & $<0.001$ \\
\hline Vaginal & $175(37 \%)$ & $44(34 \%)$ & $106(36 \%)$ & $25(54 \%)$ & \\
\hline Unknown & $14(3 \%)$ & $5(4 \%)$ & $4(2 \%)$ & $5(11 \%)$ & \\
\hline \multicolumn{6}{|l|}{ 1-minute APGAR score } \\
\hline$<=2$ & $130(30 \%)$ & $38(31 \%)$ & $88(31 \%)$ & $4(11 \%)$ & $<0.001$ \\
\hline 3 to 6 & $214(49 \%)$ & $59(49 \%)$ & $143(51 \%)$ & $12(33 \%)$ & \\
\hline$>=7$ & $92(21 \%)$ & $24(20 \%)$ & $48(18 \%)$ & $20(56 \%)$ & \\
\hline \multicolumn{6}{|l|}{ 5-minute APGAR score } \\
\hline$<=2$ & $23(5 \%)$ & $5(4 \%)$ & $16(6 \%)$ & $2(6 \%)$ & 0.059 \\
\hline 3 to 6 & $143(33 \%)$ & $41(34 \%)$ & $98(35 \%)$ & $4(11 \%)$ & \\
\hline$>=7$ & $270(62 \%)$ & $75(62 \%)$ & $165(59 \%)$ & $30(83 \%)$ & \\
\hline Prenatal diagnosis & $171(37 \%)$ & $55(43 \%)$ & $98(33 \%)$ & $18(39 \%)$ & 0.191 \\
\hline $\begin{array}{l}\text { Proportion of prenatally } \\
\text { diagnosed cases who were inborn }\end{array}$ & $41(24 \%)$ & $9(16 \%)$ & $25(25 \%)$ & $7(39 \%)$ & 0.131 \\
\hline \multicolumn{6}{|l|}{ Primary reason for admission } \\
\hline Anomalies or syndrome & $190(41 \%)$ & $32(25 \%)$ & $136(47 \%)$ & $22(48 \%)$ & $<0.001$ \\
\hline Respiratory & $87(19 \%)$ & $56(43 \%)$ & $29(10 \%)$ & $2(4 \%)$ & $<0.001$ \\
\hline Cardiac & $81(17 \%)$ & $14(11 \%)$ & $57(20 \%)$ & $10(22 \%)$ & 0.068 \\
\hline Preterm birth & $60(13 \%)$ & $11(8 \%)$ & $45(15 \%)$ & $4(9 \%)$ & 0.102 \\
\hline Surgical evaluation & $34(7 \%)$ & $11(8 \%)$ & $15(5 \%)$ & $8(17 \%)$ & 0.010 \\
\hline
\end{tabular}

$I Q R$ interquartile range.

Percentages are column percentages.

$p$ values of $<0.05$ are in bold font.

${ }^{\mathrm{a}}$ Contain missing values and numbers do not add up to $100 \%$.

was not statistically different between groups. Prematurity ( $<32$ weeks) was more common in the group receiving intensive medical treatment.

Appendix B (online) shows the types of medical interventions received by infants in the 3 groups. One third of infants were intubated in the delivery room, but cardiac compressions or epinephrine use was infrequent. Infants who underwent surgeries were more likely to be mechanically ventilated and required longer duration of mechanical ventilation. The overall proportion of infants who received inotropic support was small.

Appendix C (online) lists the most common surgeries performed in the NICU and outcomes for those patients. For selected surgeries, we also list the total number of patients with associated anomalies in the cohort. For example, for 
infants who receive a tracheoesophageal fistula ligation, we list the number of patients in the cohort with a tracheoesophageal fistula. Surgical interventions were performed in $25 \%$ of patients. The most frequent surgeries were gastrostomy placement and bronchoscopy, but a small proportion of patients underwent major cardiac, thoracic, or neurological surgeries. When we looked at the number of surgeries per patient, $53(41 \%)$ patients had 1 surgery performed, 48 (37\%) had 2-3 surgeries performed, and 28 (22\%) patients had $>4$ surgeries performed.

Table 2 shows discharge outcomes for infants by treatment group. Overall survival to NICU discharge was $40 \%$ (when including infants who were transferred in the denominator). When excluding infants who were transferred out from the denominator of total ( $n=419$ infants since 48 were transferred), the survival to NICU discharge was $45 \%$. Infants in the non-intensive treatment group were most likely to survive, while infants in the intensive medical treatment group were least likely to survive. Most infants who died in intensive treatment groups had withdrawal of life-sustaining therapies and/or do not resuscitate (DNR) orders in place at the time of death. All infants in the nonintensive care group had DNR orders in place at the time of death. Among survivors, infants who received surgical interventions had a longer length of stay (LOS), greater duration of mechanical ventilation, and were more likely to be discharged home with feeding and respiratory medical support. More than half of the infants discharged home were referral to palliative care. Infants who received surgical interventions were less likely to be referred to palliative care.

Figure 1 shows temporal trends in admissions of infants with T13 and T18 from 2010 to 2016 separated by treatment group. The total number of infants admitted with T13 or T18 increased over the study period $(p=0.002)$ as did the proportion of total admissions who received surgical interventions $(p=0.003)$, from $15 \%$ in 2010 to $33 \%$ in 2016. During the same time period, the number of centers contributing to $\mathrm{CHNC}$ also increased from 22 centers in 2010 to 30 centers in 2016. The number of infants who received intensive treatment or non-intensive treatment did not statistically increase over time. During the same study period, the proportion of infants who survived overall, or by treatment group did not significantly change.

Figure 2 demonstrates inter-center variability in admissions for infants with T13 or T18 as well as the proportion of infants in the three groups for centers admitting $>10$ total patients. There was wide variability in both number of admissions and proportion of infants receiving medical and surgical treatment across centers. The proportion of infants receiving intensive medical interventions ranged from 0 to $90 \%$, with a strong positive correlation between center volume and patients who received intensive medical treatment $(r=0.89, p<0.001)$ as well as those receiving surgical treatment $(r=0.5, p=0.003)$.

Table 3 compares the characteristics of infants who died vs. survived in each of the treatment groups. In both intensive treatment groups, infants who died were more likely to be preterm, of lower birth weight, male, require intubation or mechanical ventilation, and receive systemic steroids for cardiovascular support. In the intensive medical treatment group, the infants who died were more likely to be admitted for a surgical evaluation. In the non-intensive treatment group, the majority of infants who died were admitted for major anomalies. In a multivariable regression model (Appendix D (online)) for both intensive treatment groups, male sex, low birth weight, and need for mechanical ventilation ( $>1$ week) were all significantly associated with mortality. Infants who died in both intensive treatment groups spent twice the amount of time on mechanical ventilation as infants who survived.

\section{Discussion}

This is the first study to describe medical and surgical interventions and how they impacted outcomes of infants with T13 or T18 admitted to a large multi-center collaborative of children's hospital NICUs. Our primary conclusions are as follows:

(1) The majority of infants with T13 or T18 admitted to CHNC NICUs received intensive medical or surgical treatment. This cohort likely represents a selected sample of infants for whom families may have desired at least an initial NICU evaluation and a trial of intensive care, and/or those in whom a confirmatory diagnosis was not achieved prior to NICU admission.

(2) The most common surgery performed in the NICU was gastrostomy tube placement, but some infants underwent more complex surgeries.

(3) Overall survival to NICU discharge was 40\%; survival was $72 \%$ for infants in the non-intensive treatment group; $51 \%$ in the intensive surgical treatment group, and $32 \%$ in the intensive medical treatment group.

(4) Infants who died after receiving intensive treatment were more likely male, of low birth weight, and received prolonged mechanical ventilation compared to those who survived. Although numbers were small, certain surgeries were associated with high mortality.

(5) There was wide inter-center variability in total number of admissions of infants with T13 or T18 as well as the proportion of infants receiving intensive treatment by center. 
Table 2 Hospital Outcomes for Infants with T13 or T18 who received surgical, intensive medical or non-intensive treatment $(n=467)$.

\begin{tabular}{|c|c|c|c|c|c|}
\hline & $\begin{array}{l}\text { Entire Cohort } \\
467\end{array}$ & $\begin{array}{l}\text { Surgical } \\
\text { treatment } \\
129\end{array}$ & $\begin{array}{l}\text { Intensive medical } \\
\text { treatment } \\
292\end{array}$ & $\begin{array}{l}\text { Non-intensive } \\
\text { treatment } \\
46\end{array}$ & $p$-value \\
\hline Died before hospital discharge & 231 (49) & $42(32)$ & $179(61)$ & $10(22)$ & $<0.001$ \\
\hline \multicolumn{6}{|l|}{ Of those who died, } \\
\hline DNR Orders & $175(76)$ & $32(76)$ & $133(74)$ & $10(100)$ & 0.182 \\
\hline Medical care withdrawn & $164(71)$ & $34(81)$ & $129(72)$ & $1(10)$ & $<0.001$ \\
\hline LOS, median (IQR) & $7[3,17]$ & $17[9,47]$ & $7[3,15]$ & $2[0,3]$ & $<0.001$ \\
\hline Survived to hospital discharge & $188(40)$ & $66(51)$ & $89(30)$ & $33(72)$ & $<0.001$ \\
\hline \multicolumn{6}{|l|}{ Of those who survived to discharge, } \\
\hline LOS, median (IQR) & $13[6,25]$ & $27[16,43]$ & $10[6,17]$ & $6[3,12]$ & $<0.001$ \\
\hline Total ventilator days, median (IQR) & $5[2,10]$ & $6[4,18]$ & $3[1,5]$ & . [.,..] & 0.003 \\
\hline \multicolumn{6}{|l|}{ Therapy at discharge } \\
\hline \multicolumn{6}{|l|}{ Feeding support } \\
\hline Gastrostomy tube feedings & $41(22)$ & $41(62)$ & $0(0)$ & $0(0)$ & $<0.001$ \\
\hline \multicolumn{6}{|l|}{ Respiratory support at discharge } \\
\hline Tracheostomy & $5(3)$ & $5(8)$ & $0(0)$ & $0(0)$ & 0.009 \\
\hline Apnea monitor & $66(35)$ & $28(42)$ & $31(35)$ & $7(21)$ & 0.241 \\
\hline Home oxygen & $92(49)$ & $34(51)$ & $51(57)$ & $7(21)$ & 0.002 \\
\hline Mechanical ventilator & $5(3)$ & $4(6)$ & $1(1)$ & $0(0)$ & 0.097 \\
\hline \multicolumn{6}{|l|}{ Route of feeds at discharge } \\
\hline Breast & $20(11)$ & $3(4)$ & $9(10)$ & $8(24)$ & 0.026 \\
\hline Bottle & $51(27)$ & $10(15)$ & $25(28)$ & $16(48)$ & 0.005 \\
\hline Tube feedings & $157(83)$ & $62(94)$ & $75(84)$ & $20(61)$ & 0.001 \\
\hline Gastrostomy tube feedings & $41(22)$ & $41(66)$ & $0(0)$ & $0(0)$ & \\
\hline Nasogastric/orogastric tube feedings & $113(60)$ & $18(27)$ & $75(84)$ & $19(58)$ & \\
\hline Transpyloric feeds & $3(1)$ & $2(3)$ & $0(0)$ & $1(3)$ & \\
\hline $\begin{array}{l}\text { Discharged with a referral to palliative } \\
\text { care }^{\mathrm{a}}\end{array}$ & 97/177(55\%) & $22 / 64(34 \%)$ & $54 / 83(65 \%)$ & $21 / 30(70 \%)$ & $<0.001$ \\
\hline Transferred out ${ }^{\mathrm{b}}$ & $48(10)$ & $21(16)$ & $24(8)$ & $3(6)$ & 0.029 \\
\hline LOS median (IQR) & $17[6,35]$ & $35[19,68]$ & $8[2,19]$ & $2[0,7]$ & $<0.001$ \\
\hline \multicolumn{6}{|c|}{ Of those who transferred out, reasons for transfer, } \\
\hline Chronic care & $1(2)$ & $1(5)$ & $0(0)$ & $0(0)$ & 0.041 \\
\hline $\begin{array}{l}\text { Growth/discharge planning/ } \\
\text { continuing care }\end{array}$ & $17(35)$ & $7(33)$ & $8(33)$ & $2(67)$ & \\
\hline Medical/diagnostic services & $1(2)$ & $1(5)$ & $0(0)$ & $0(0)$ & \\
\hline Palliative care & $18(37)$ & $3(14)$ & $14(58)$ & $1(33)$ & \\
\hline Surgery & $10(21)$ & $9(43)$ & $1(4)$ & $0(0)$ & \\
\hline Unknown & $1(2)$ & $0(0)$ & $1(4)$ & $0(0)$ & \\
\hline
\end{tabular}

$D N R$ do not resuscitate, $I Q R$ interquartile range, $L O S$ length of stay.

$p$ values of $<0.05$ are in bold font.

${ }^{\mathrm{a}}$ Contains 11 missing values.

${ }^{\mathrm{b}}$ The number of infants transferred out $(n=48)$ is included in the denominator for percentages.

Overall, this cohort admitted to CHNC NICUs likely represents a select group of infants referred for advanced care. Their families may have had prenatal suspicion for these conditions and desired interventions, or only discovered the diagnosis postnatally. This cohort does not encapsulate infants who were cared for in newborn nurseries or who died in the delivery room. Prior studies show that fewer than $5 \%$ of parents who receive a prenatal diagnosis of $\mathrm{T} 13$ or $\mathrm{T} 18$ choose to receive intensive medical interventions in the NICU; most choose to terminate the pregnancy or to deliver with a plan for comfort care [9]. We cannot tell from our data the families' goals for NICU 
Fig. 1 Time trends in NICU admissions, number of infants receiving intensive vs. nonintensive treatment and percentage of surviving infants from 2010-16 $(n=467)$. X-axis represents year and number of centers contributing data to the $\mathrm{CHNC}$ in that year in parenthesis. Y-axis represents number of $\mathrm{T} 13$ or $\mathrm{T} 18$ admissions in that year.
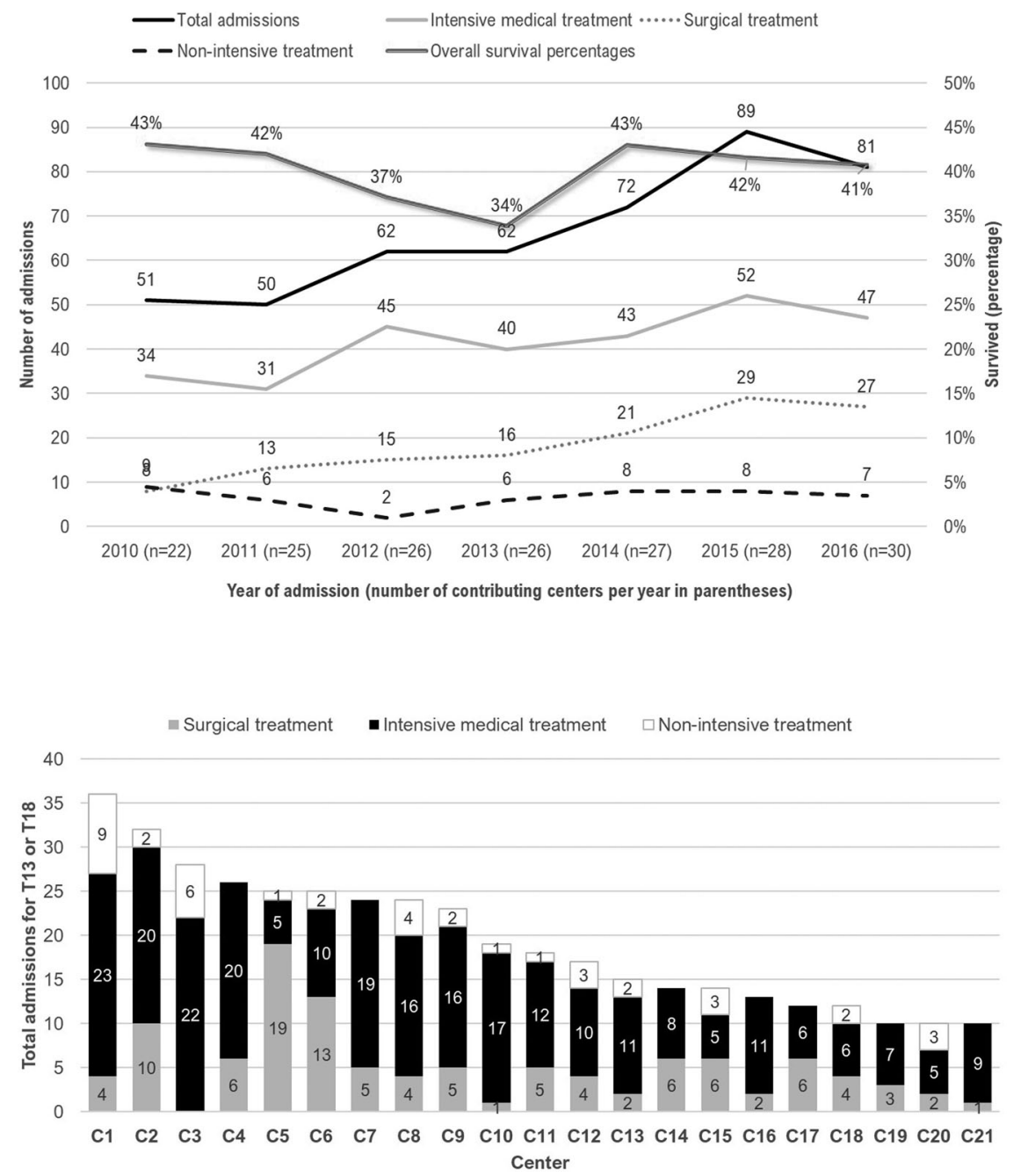
Center
Fig. 2 Center variation in 3 treatment groups for infants with $\mathrm{T} 13$ or $\mathrm{T} 18$ in centers with $>10$ patients $(n=407)$. $\mathrm{C} 1$ through $\mathrm{C} 21$ denote different center locations. admission; whether for comfort care alone, a trial of interventions, or intensive treatment followed by redirection of care to comfort. It is somewhat surprising in the modern era of prenatal testing that only a third of infants in this cohort had a prenatal diagnosis of T13 or T18. However, when we examined trends in the proportion of prenatally diagnosed cases by year, a significantly higher number of cases $(53 \%$ in 2016 vs. $17 \%$ in $2010, p<0.001$ ) were diagnosed prenatally in later years. Those without confirmatory prenatal diagnosis may have had suspicion based on ultrasound screening or non-invasive screening tests and were either not offered or refused additional prenatal confirmatory genetic testing. As a result, some infants may have received intensive interventions initially followed by realignment of goals of care to comfort after a postnatal genetic diagnosis.

Infants who received non-intensive NICU care may represent a select group whose care focused on comfort or, a physiologically more stable population who did not require many interventions. Fewer in this group were born preterm or admitted for respiratory reasons. Yet among infants in this group who died in the NICU, the majority had anomalies, perhaps leading to families choosing a plan for comfort care. Infants who received non-intensive treatment were more likely to be discharged home after a short NICU stay, and also more likely to be feeding by bottle or breast. These results support earlier studies that show that some infants with T13 and 18 receive significant NICU interventions, while others infants may not necessarily require a lot of early interventions [2].

The most common surgery performed on infants admitted in our cohort was gastrostomy placement, but some infants did undergo major cardiac, neurologic, and thoracic surgeries. The next most common procedure was a bronchoscopy, presumably for infants with stridor or respiratory distress, which are reportedly common, or in infants with extubation failure [11, 12]. Infants who underwent major cardiac surgeries are likely underrepresented in this study, because the CHND does not capture data on infants cared for exclusively in cardiac ICUs. While surgeries such as diaphragmatic hernia repair were rare, they did occur. In some patients, multiple surgeries were performed. Nelson et al. evaluated types of surgeries performed in a population- 
Table 3 Characteristics of Infants who survived vs. died in the 3 treatment groups $(n=467)$.

\begin{tabular}{|c|c|c|c|c|c|c|c|c|c|}
\hline & \multicolumn{3}{|c|}{ Surgical treatment } & \multicolumn{3}{|c|}{ Intensive medical treatment } & \multicolumn{3}{|c|}{ Non-intensive treatment } \\
\hline & $\begin{array}{l}\text { Survived }^{\mathrm{a}} \\
87\end{array}$ & $\begin{array}{l}\text { Died } \\
42\end{array}$ & $p$-value & $\begin{array}{l}\text { Survived }^{\mathrm{a}} \\
113\end{array}$ & $\begin{array}{l}\text { Died } \\
179\end{array}$ & $p$-value & $\begin{array}{l}\text { Survived }^{\mathrm{a}} \\
36\end{array}$ & $\begin{array}{l}\text { Died } \\
10\end{array}$ & $p$-value \\
\hline \multicolumn{10}{|l|}{ Gestational age at birth (weeks) } \\
\hline$\leq 27+6 / 7$ & $1(1)$ & $1(2)$ & 0.028 & $0(0)$ & $3(2)$ & $<0.001$ & $0(0)$ & $0(0)$ & 0.054 \\
\hline 28 to $31+6 / 7$ & $2(2)$ & $5(12)$ & & $3(3)$ & $29(16)$ & & $0(0)$ & $2(20)$ & \\
\hline 32 to $36+6 / 7$ & $33(38)$ & $20(48)$ & & $23(20)$ & $71(40)$ & & $6(17)$ & $1(10)$ & \\
\hline$\geq 37$ & $51(59)$ & $16(38)$ & & $87(77)$ & $76(42)$ & & $30(83)$ & $7(70)$ & \\
\hline \multicolumn{10}{|l|}{ Birth weight $(\mathrm{g})^{\mathrm{b}}$} \\
\hline$<=1500 \mathrm{~g}$ & $14(16)$ & $11(26)$ & 0.023 & $12(11)$ & $57(32)$ & $<0.001$ & $3(8)$ & $3(30)$ & 0.247 \\
\hline $1501-2499 \mathrm{~g}$ & $52(61)$ & $29(69)$ & & $67(60)$ & $83(47)$ & & $23(66)$ & $5(50)$ & \\
\hline$>=2500 \mathrm{~g}$ & $19(22)$ & $2(5)$ & & $33(29)$ & $36(20)$ & & $9(26)$ & $2(20)$ & \\
\hline Male gender & $26(30)$ & $26(62)$ & 0.001 & $33(29)$ & $90(50)$ & $<0.001$ & $9(25)$ & $6(60)$ & 0.057 \\
\hline \multicolumn{10}{|l|}{ Maternal race ${ }^{b}$} \\
\hline Asian & $1(1)$ & $0(0)$ & 0.954 & $2(2)$ & $7(4)$ & 0.113 & & & 0.736 \\
\hline Black & $24(28)$ & $11(26)$ & & $23(20)$ & $50(28)$ & & $9(25)$ & $2(20)$ & \\
\hline Native Hawaiian/Pacific Islander & $0(0)$ & $0(0)$ & & $2(2)$ & $0(0)$ & & $2(5)$ & $0(0)$ & \\
\hline Other & $11(13)$ & $6(14)$ & & $13(11)$ & $28(16)$ & & $6(17)$ & $1(10)$ & \\
\hline White & $44(51)$ & $23(55)$ & & $68(60)$ & $84(47)$ & & $15(42)$ & $7(70)$ & \\
\hline \multicolumn{10}{|l|}{ Primary reason for admission } \\
\hline Anomalies or Syndrome & $20(23)$ & $12(29)$ & 0.519 & $52(46)$ & $84(47)$ & 0.905 & $13(36)$ & $9(90)$ & 0.004 \\
\hline Cardiac & $11(13)$ & $3(7)$ & 0.547 & $25(22)$ & $32(18)$ & 0.449 & $9(25)$ & $1(10)$ & 0.420 \\
\hline Preterm birth & $4(5)$ & $1(2)$ & 1.000 & $0(0)$ & $10(6)$ & NA & $0(0)$ & $0(0)$ & NA \\
\hline Respiratory & $9(10)$ & $2(5)$ & 0.502 & $23(20)$ & $22(12)$ & 0.069 & $4(11)$ & $0(0)$ & NA \\
\hline Surgical evaluation & $33(38)$ & $23(55)$ & 0.089 & $6(5)$ & $23(13)$ & 0.044 & $2(5)$ & $0(0)$ & NA \\
\hline Other & $10(11)$ & $1(2)$ & 0.102 & $7(6)$ & $8(4)$ & 0.590 & $8(22)$ & $0(0)$ & NA \\
\hline \multicolumn{10}{|l|}{ NICU interventions ${ }^{\mathrm{c}}$} \\
\hline \multicolumn{10}{|l|}{ Delivery room interventions } \\
\hline PPV & $43(57)$ & $26(67)$ & 0.322 & $82(74)$ & $133(78)$ & 0.566 & $0(0)$ & $0(0)$ & NA \\
\hline Intubation & $17(21)$ & $22(56)$ & $<0.001$ & $25(23)$ & $95(55)$ & $<0.001$ & $0(0)$ & $0(0)$ & NA \\
\hline Chest compressions & $4(5)$ & $3(7)$ & 0.686 & $7(6)$ & $22(13)$ & 0.109 & $0(0)$ & $0(0)$ & NA \\
\hline Epinephrine & $1(1)$ & $1(2)$ & 1.000 & $0(0)$ & $5(3)$ & NA & $0(0)$ & $0(0)$ & NA \\
\hline None of the above & $43(49)$ & $11(26)$ & 0.014 & $27(24)$ & $37(21)$ & 0.562 & $36(100)$ & $10(100)$ & 0.171 \\
\hline \multicolumn{10}{|l|}{ Respiratory support during admission } \\
\hline Conventional ventilation & $67(82)$ & $41(98)$ & 0.011 & $40(37)$ & $140(78)$ & $<0.001$ & $0(0)$ & $0(0)$ & NA \\
\hline HFJV or HFOV & $3(4)$ & $11(26)$ & $<0.001$ & $3(3)$ & $40(22)$ & $<0.001$ & $0(0)$ & $0(0)$ & NA \\
\hline NIMV & $21(26)$ & $5(12)$ & 0.103 & $12(11)$ & $23(13)$ & 0.714 & $0(0)$ & $0(0)$ & NA \\
\hline NCPAP & $32(39)$ & $7(17)$ & 0.014 & $36(34)$ & $40(22)$ & 0.039 & $0(0)$ & $0(0)$ & NA \\
\hline High flow nasal cannula & $45(55)$ & $17(40)$ & 0.184 & $65(61)$ & $56(31)$ & $<0.001$ & $0(0)$ & $0(0)$ & NA \\
\hline iNO & $1(1)$ & $3(7)$ & 0.112 & $1(1)$ & $23(13)$ & $<0.001$ & $0(0)$ & $0(0)$ & NA \\
\hline None of the above & $8(9)$ & $0(0)$ & NA & $18(16)$ & $0(0)$ & NA & $36(100)$ & $10(100)$ & 0.171 \\
\hline $\begin{array}{l}\text { Ventilator days in those receiving invasive mechanical } \\
\text { ventilation, median (IQR) days }\end{array}$ & $8[4,21]$ & $19[8,48]$ & 0.064 & $3[1,5]$ & $6[3,11]$ & 0.001 &.$[.,]$. &.$[\ldots,]$. & NA \\
\hline \multicolumn{10}{|l|}{ Cardiovascular interventions } \\
\hline Vasopressors/Inotropic support & $0(0)$ & $1(4)$ & NA & $2(5)$ & $20(16)$ & 0.107 & $0(0)$ & $0(0)$ & NA \\
\hline Surfactant & $7(8)$ & $6(15)$ & 0.348 & $15(13)$ & $38(22)$ & 0.086 & $0(0)$ & $0(0)$ & NA \\
\hline Systemic steroids & $3(13)$ & $7(50)$ & 0.023 & $0(0)$ & $27(75)$ & NA & $0(0)$ & $0(0)$ & NA \\
\hline None of the above & $77(88)$ & $29(69)$ & 0.013 & $98(87)$ & $118(66)$ & $<0.001$ & $36(100)$ & $10(100)$ & 0.171 \\
\hline
\end{tabular}

HFJV high frequency jet ventilation, HFOV high frequency oscillatory ventilation, NIMV non-invasive mechanical ventilation, NCPAP nasal continuous positive airway pressure, $i N O$ inhaled nitric oxide, $I Q R$ interquartile range.

$p$-values of $<0.05$ are in bold font.

${ }^{\mathrm{a}}$ The survived group includes infants who were transferred.

${ }^{\mathrm{b}}$ Contains missing values and totals do not add up to $100 \%$.

${ }^{\mathrm{c}}$ Some infants may have received more than one intervention and totals may add up to $>100 \%$. 
based study in Canada and found that ear-nose-throat surgeries followed by feeding ostomies were the most common procedures in these children [3]. Many ear-nose-throat procedures, such as cleft palate repair, are not typically performed in the neonatal period, which may be why gastrostomy was more common in our cohort. In addition, a gastrostomy tube can provide feeding stability for an infant with T13 or T18 and facilitate discharge home. Our finding that infants undergoing surgical procedures will spend more time ventilated and have a longer NICU length of stay can be used to counsel families providing more accurate expectations of their child's hospital course.

Survival to NICU discharge was $40 \%$, similar to what has been reported previously in the NICU population [2]. Survival in the group that received surgical treatment was higher than in the group that received intensive medical treatment alone. This may be because the most common surgeries performed were ones not necessarily associated with high mortality (such as gastrostomy tubes). It is also possible that some infants in the intensive medical treatment group had major surgical anomalies but did not undergo surgery because they were too sick, or surgery did not align with parental goals. Infants in the nonintensive treatment group were most likely to survive and were discharged earlier than other groups. This could be due to one of two reasons; the infants were physiologically more stable and did not require a lot of interventions, or families chose to take infants home sooner with the goals of comfort care and hospice support. This information may be useful for families considering varying levels of intervention for their child: survival after medical and surgical interventions is possible, but these infants will have a longer hospital stay and will need more support to discharge home. On the other hand, there are infants who do not necessarily require a lot of interventions, and families who choose to limit intensive interventions may still be able to achieve the goal of taking their baby home depending on the child's physiologic stability.

We found that the majority of infants who died in the intensive treatment groups had withdrawal of life-sustaining therapies; this may have been due to realignment of goals of care to comfort after interventions 'failed' or after confirmatory postnatal testing. We are not able to tell from this data what conversations were had with families when interventions were started or when the decision was made to stop. It is notable that the frequency of death due to withdrawal of life-sustaining therapies in this cohort $(71 \%)$ is similar to the rate reported in the entire population of infants who die in CHNC NICUs [13].

Our results additionally show that there is wide variability in the numbers of infants admitted to US children's hospitals with $\mathrm{T} 13$ or T18, as well as the proportion of those infants who received intensive vs. non-intensive treatment. Differences in intensive treatment rates may to some degree represent differences between what individual centers offer to families. For families whose children may need intensive interventions and who desire such interventions, especially surgical, it will be important to determine which children will benefit from surgical interventions and in whom the interventions may cause harm. Many recent studies, including ours, focus on short-term outcomes, and we do not know the impact of interventions on long-term survival or other developmental outcomes. Indeed, ethical arguments have been made both for and against why these children should or should not be offered certain surgeries [14].

Previous studies have shown that male sex, lower birth weight, and need for mechanical ventilation are predictors of mortality [2]. Our data extend these findings for children receiving both medical and surgical interventions. Even among infants who received minimal interventions, male infants were at higher risk of dying. The number of infants undergoing more complex surgeries such as diaphragmatic hernia repair was small, but these surgeries did occur, and mortality was high. Before offering or recommending an intervention, it is important to understand parents' goals and how they expect the intervention would benefit their child. For example, some families may desire tube placement for long-term nutrition but would not want major cardiac surgeries. Families may be given the option of taking their baby home with a nasogastric tube for the short term, with the option of a gastrostomy tube later if the family desires. Other families may demand all interventions that a child without T13 or T18 would be offered, even if likelihood of long-term survival is low.

We do not have information on long-term survival or developmental outcomes for our cohort. While ethical arguments regarding what interventions should and should not be offered to families is beyond the scope of this paper, we urge providers to engage with families in open and honest discussion about the risks and benefits of interventions and the limits of our current knowledge. Decision-making should continue to be personalized, through a process of partnership with families. The significant mortality observed in the small number of these infants undergoing complex surgeries calls for caution in wider prescription of these procedures and continued discussion of the burdens and potential benefits of such procedures.

\section{Limitations}

This was a large multi-center study reporting the outcome of infants with T13 or T18 in the CHNC consortium. For individual patients in our database, we do not know what the goals of care were at the time of birth, or when in the course of care decisions regarding DNR or withdrawal were made. Treatment group does not necessarily imply intention behind the treatment, especially for cases of postnatal diagnosis. We cannot ascertain nuanced details of prenatal or postnatal counseling that families received nor if families perceived that interventions may have benefited their children regardless of survival. 
Surgical treatment encompassed a wide range of interventions with varying morbidity and mortality risks; overall survival numbers may not apply to more complex surgeries. Cardiac surgeries are underrepresented in this cohort. Additionally, we were not able to assess long-term outcomes in this cohort.

\section{Conclusions}

Infants with T13 or T18 admitted to CHNC NICUs underwent a variety of interventions during their initial hospital course. Survival to NICU discharge was possible after medical and surgical interventions, but surviving infants had a longer hospital stay and needed more discharge supports. This study provides a better understanding of the spectrum of NICU care T13 and T18 infants receive and can be used to improve future counseling to families that desire interventions.

Acknowledgements We are indebted to the following institutions that serve the infants and their families, and these institutions also have invested in and continue to participate in the Children's Hospital's Neonatal Database (CHND). Jeanette Asselin, Beverly Brozanski, David Durand (ex officio), Francine Dykes (ex officio), Jacquelyn Evans (Executive Director), Theresa Grover, Karna Murthy (Chair), Michael Padula, Eugenia Pallotto, Anthony Piazza, Kristina Reber, and Billie Short are members of the Children's Hospitals Neonatal Consortium, Inc. For more information, please contact: support@ thechnc. org. The site sponsors/contributors for the CHND include:

Palliative Care and Ethics Focus Group of the Children's Hospital Neonatal Consortium (CHNC) Anthony Piazza ${ }^{35}$, Gregory Sysyn ${ }^{36}$, Carl Coghill $^{37}$, Ajay Talati ${ }^{38}$, Anne Hansen ${ }^{39}$, Tanzeema Houssain ${ }^{39}$, Karna Murthy $^{40}$, Gustave Falciglia ${ }^{40}$, Beth Haberman ${ }^{41}$, Kristina Reber ${ }^{42}$, Rashmin Savani ${ }^{43}$, Theresa Grover ${ }^{44}$, Girija Natarajan ${ }^{45}$, Annie $\mathrm{Chi}^{46}$, Yvette Johnson ${ }^{46}$, Gautham Suresh ${ }^{47}$, William Engle ${ }^{48}$, Eugenia Pallotto $^{49}$, Robert Lyle ${ }^{50}$, Becky Rogers ${ }^{50}$, Rachel Chapman ${ }^{51}$, Jamie Limjoco $^{52}$, Priscilla Joe ${ }^{53}$, Jacquelyn Evans ${ }^{54}$, Michael Padula ${ }^{54}$, David Munson ${ }^{54}$, Suzanne Touch ${ }^{55}$, Beverly Brozanski ${ }^{56}$, Rakesh Rao ${ }^{57}$, Amit Mathur $^{57}$, Victor McKay ${ }^{58}$, Mark Speziale ${ }^{59}$, Laurel Moyer ${ }^{59}$, Billie Short $^{60}$, Kevin Sullivan ${ }^{61}$, Con Yee Ling ${ }^{62}$, Michael Uhing ${ }^{63}$, Lynne Willett $^{64}$, Nicole Birge ${ }^{64}$, Rajan Wadhawan ${ }^{65}$, Elizabeth JacobsenMisbe $^{66}$, Robert DiGeronimo ${ }^{66}$, Kyong-Soon Lee ${ }^{67}$, Michel Mikhael ${ }^{68}$

${ }^{35}$ Children's Healthcare of Atlanta, Atlanta, GA, USA; ${ }^{36}$ Children's Healthcare of Atlanta at Scottish Rite, Atlanta, USA; ${ }^{37}$ Children's Hospital of Alabama, Birmingham, AL, USA; ${ }^{38}$ Le Bonheur Children's Hospital, Memphis, TN, USA; ${ }^{39}$ Children's Hospital Boston, Boston, MA, USA; ${ }^{40}$ Ann \& Robert H. Lurie Children's Hospital of Chicago, Chicago, IL, USA; ${ }^{41}$ Cincinnati Children's Hospital, Cincinnati, OH, USA; ${ }^{42}$ Nationwide Children's Hospital, Columbus, OH, USA; ${ }^{43}$ Children's Medical Center, Dallas, TX, USA; ${ }^{44}$ Children's Hospital Colorado, Aurora, CO, USA; ${ }^{45}$ Children's Hospital of Michigan, Detroit, MI, USA; ${ }^{46}$ Cook Children's Health Care System, Fort Worth, TX, USA; ${ }^{47}$ Texas Children's Hospital, Houston, TX, USA; ${ }^{48}$ Riley Children's Hospital, Indianapolis, IN, USA; ${ }^{49}$ Children's Mercy Hospitals \& Clinics, Kansas City, MO, USA; ${ }^{50}$ Arkansas Children's Hospital, Little Rock, AR, USA; ${ }^{51}$ Children's Hospital Los Angeles, Los Angeles, CA, USA; ${ }^{52}$ American Family Children's Hospital, Madison, WI, USA; ${ }^{53} \mathrm{Chil}-$ dren's Hospital \& Research Center Oakland, Oakland, CA, USA; ${ }^{54}$ The Children's Hospital of Philadelphia, Philadelphia, PA, USA;
${ }^{55}$ St. Christopher's Hospital for Children, Philadelphia, PA, USA; ${ }^{56}$ Children's Hospital of Pittsburgh of UPMC, Pittsburgh, PA, USA; ${ }^{57}$ St. Louis Children's Hospital, St Louis, MO, USA; ${ }^{58}$ All Children's Hospital, St, Petersburg, FL, USA; ${ }^{59}$ Rady Children's Hospital, San Diego, CA, USA; ${ }^{60}$ Children's National Medical Center, Washington, DC, USA; ${ }^{61} \mathrm{AI}$ DuPont Hospital for Children, Wilmington, DE, USA; ${ }^{62}$ Primary Children's Medical Center, Salt Lake City, UT, USA; ${ }^{63}$ Children's Hospital of Wisconsin, Milwaukee, WI, USA; ${ }^{64}$ Children's Hospital of Omaha, Omaha, NE, USA; ${ }^{65}$ Florida Hospital for Children, Orlando, FL, USA; ${ }^{66}$ Seattle Children's Hospital, Seattle, WA, USA; ${ }^{67}$ Hospital for Sick Children, Toronto, ON, USA; ${ }^{68}$ Children's Hospital Orange County, Los Angeles, CA, USA

\section{Compliance with ethical standards}

Conflict of interest The authors declare no competing interests.

Publisher's note Springer Nature remains neutral with regard to jurisdictional claims in published maps and institutional affiliations.

\section{References}

1. Nelson KE, Hexem KR, Feudtner C. Inpatient Hospital Care of Children With Trisomy 13 and Trisomy 18 in the United States. Pediatrics 2012;129:869-76.

2. Acharya K, Leuthner S, Clark R, Nghiem-Rao TH, Spitzer A, Lagatta J. Major anomalies and birth-weight influence NICU interventions and mortality in infants with trisomy 13 or 18 . J Perinatol. 2017;37:420-6.

3. Nelson KE, Rosella LC, Mahant S, Guttmann A. Survival and surgical interventions for children with trisomy 13 and 18. JAMA. 2016;316:420.

4. Pallotto I, Lantos JD. Treatment decisions for babies with trisomy 13 and 18. HEC Forum. 2017;29:213-22.

5. Guon J, Wilfond BS, Farlow B, Brazg T, Janvier A. Our children are not a diagnosis: the experience of parents who continue their pregnancy after a prenatal diagnosis of trisomy 13 or 18 . Am J Med Genet Part A. 2014;164:308-18.

6. Leuthner SR, Acharya K. Perinatal counseling following a diagnosis of trisomy 13 or 18: incorporating the facts, parental values, and maintaining choices. Adv Neonatal Care. 2020;20:204-15.

7. Subramaniam A, Jacobs AP, Tang Y, Neely C, Philips JB, Biggio JR, et al. Trisomy 18: a single-center evaluation of management trends and experience with aggressive obstetric or neonatal intervention. Am J Med Genet. 2016;170:838-46.

8. Janvier A, Farlow B, Barrington KJ. Parental hopes, interventions, and survival of neonates with trisomy 13 and trisomy 18. Am J Med Genet Part C: Semin Med Genet. 2016;172:279-87.

9. Winn P, Acharya K, Peterson E, Leuthner S. Prenatal counseling and parental decision-making following a fetal diagnosis of trisomy 13 or 18. J Perinatol. 2018;38:788-96.

10. Murthy K, Dykes FD, Padula MA, Pallotto EK, Reber KM, Durand DJ, et al. The Children's Hospitals Neonatal Database: an overview of patient complexity, outcomes and variation in care. $\mathrm{J}$ Perinatol. 2014;34:582-6.

11. Billings KR, Rastatter JC, Lertsburapa K, Schroeder JW Jr. An analysis of common indications for bronchoscopy in neonates and findings over a 10-year period. JAMA Otolaryngol-Head Neck Surg. 2015;141:112-9.

12. Swanson SK, Schumacher KR, Ohye RG, Zampi JD. Impact of trisomy 13 and 18 on airway anomalies and pulmonary complications after cardiac surgery. J Thorac Cardiovasc Surg. 2020; S0022-5223(20)32473-9. 
13. Fry JT, Matoba N, Datta A, DiGeronimo R, Coghill $\mathrm{CH}$, Natarajan G, et al. Center, gestational age, and race impact end-oflife care practices at regional neonatal intensive care units. J Pediatr. 2020;217:86-91. e1
14. Graham EM. Infants with trisomy 18 and complex congenital heart defects should not undergo open heart surgery. J Law Med Ethics. 2016;44:286-91.

\section{Affiliations}

Krishna Acharya $\oplus^{1,2} \cdot$ Steven R. Leuthner ${ }^{1,2} \cdot$ Isabella Zaniletti $\oplus^{3} \cdot$ Jason Z. Niehaus $\mathbb{D}^{4,5} \cdot$ Christine E. Bishop ${ }^{6,7}$. Carl H. Coghill (1),9 . Ankur Datta ${ }^{10,11}$ • Narendra Dereddy ${ }^{12,13}$ - Robert DiGeronimo (10 ${ }^{14,15}$ - Laura Jackson ${ }^{6,7}$. Con Yee Ling ${ }^{16,17}$ - Nana Matoba $\mathbb{1}^{10,11}$ - Girija Natarajan ${ }^{18,19}$ - Sujir Pritha Nayak ${ }^{20,21}$ - Amy Brown Schlegel ${ }^{22,23}$. Jamie Seale ${ }^{24}$ - Anita Shah ${ }^{25,26}$ • Julie Weiner ${ }^{27,28}$ • Helen O. Williams ${ }^{29,30}$ • Monica H. Wojcik $\mathbb{1 0}^{31,32}$. Jessica T. Fry $\mathbb{1}^{10,11} \cdot$ Kevin Sullivan $\mathbb{1}^{33,34}$ - on behalf of the Palliative Care and Ethics Focus Group of the Children's Hospital Neonatal Consortium (CHNC)

1 Department of Pediatrics, Medical College of Wisconsin, Milwaukee, WI, USA

2 Division of Neonatology, Children's Hospital of Wisconsin, Milwaukee, WI, USA

3 Children's Hospitals Association, Lenexa, KS, USA

4 Department of Pediatrics, Indiana University, Indianapolis, IN, USA

5 Division of Neonatology, Riley Hospital for Children, Indianapolis, IN, USA

6 Department of Pediatrics, University of Pittsburgh, Pittsburgh, PA, USA

7 Division of Newborn Medicine, UPMC Children's Hospital, Pittsburgh, PA, USA

8 Department of Pediatrics, University of Alabama at Birmingham, Birmingham, AL, USA

9 Division of Neonatology, Children's of Alabama, Birmingham, AL, USA

10 Department of Pediatrics, Northwestern University Feinberg School of Medicine, Chicago, IL, USA

11 Division of Neonatology, Ann \& Robert H. Lurie Children's Hospital of Chicago, Chicago, IL, USA

12 Department of Pediatrics, University of Central Florida, Orlando, FL, USA

13 Division of Neonatology at Advent Health for Children, Orlando, FL, USA

14 Department of Pediatrics, University of Washington, Seattle, WA, USA

15 Division of Neonatology, Seattle Children's Hospital, Seattle, WA, USA

16 Department of Pediatrics, University of Utah, Salt Lake City, UT, USA

17 Division of Neonatology at Primary Children's Hospital, Salt Lake City, UT, USA

18 Department of Pediatrics, Central Michigan University, Detroit, MI, USA
19 Division of Neonatology, Children's Hospital of Michigan, Detroit, MI, USA

20 Department of Pediatrics UT Southwestern Medical Center, Dallas, TX, USA

21 Division of Neonatology at Children's Medical Center, Dallas, TX, USA

22 Department of Pediatrics, The Ohio State College of Medicine, Columbus, OH, USA

23 Division of Neonatology, Nationwide Children's Hospital, Columbus, OH, USA

24 Intermountain Healthcare and Primary Children's Hospital, Salt Lake City, UT, USA

25 Department of Pediatrics at University of California, Irvine, CA, USA

26 Division of Neonatology, Children's Hospital of Orange County, Orange, CA, USA

27 Department of Pediatrics at University of Missouri-Kansas City School of Medicine, Kansas City, MO, USA

28 Division of Neonatology Children's Mercy - Kansas City, Kansas City, MO, USA

29 Department of Pediatrics at the Emory University School of Medicine, Atlanta, GA, USA

30 Division of Neonatology at Children's Healthcare of Atlanta, Atlanta, GA, USA

31 Divisions of Newborn Medicine and Genetics and Genomics, Department of Pediatrics, Harvard Medical School, Boston, MA, USA

32 Boston Children's Hospital, Boston, MA, USA

33 Department of Pediatrics, Sidney Kimmel Medical College at Thomas Jefferson University, Philadelphia, PA, USA

34 Division of Neonatology, Nemours/AI DuPont Hospital for Children, Wilmington, DE, USA 